\title{
Antidepressant and cognitive activities of intranasal piperine-encapsulated liposomes
}

\author{
Aroonsri Priprem $^{1}{ }^{*}$, Pennapa Chonpathompikunlert $^{2}$, Saengrawee Sutthiparinyanont ${ }^{3}$, \\ Jintanaporn Wattanathorn ${ }^{4}$
}

${ }^{1}$ Faculty of Pharmaceutical Sciences, Khon Kaen University, Khon Kaen, Thailand;

${ }^{2}$ Department of Physiology (Neuroscience Graduate Program), Faculty of Medicine, Khon Kaen University, Khon Kaen, Thailand;

${ }^{3}$ School of Pharmaceutical Sciences, University of Phayao, Phayao, Thailand;

${ }^{4}$ Department of Physiology, Faculty of Medicine, Khon Kaen University, Khon Kaen, Thailand

Email: aroonsri@kku.ac.th; bus_pennapa@yahoo.com; saengrawees@yahoo.com; jintanapornw@yahoo.com

Received 20 January 2011; revised 8 March 2011; accepted 20 March 2011.

\begin{abstract}
Antidepressant and cognitive effects of piperine-encapsulated liposomes (PL) were investigated in male Wistar rats. Oral piperine ( $5 \mathrm{mg} / \mathrm{kg}$ body weight/day) and intranasal PL (7.2 $\mu \mathrm{g} / \mathrm{day})$ were randomly assigned to daily administer for 14 days to rats which were subjected to forced swimming, Morris water maze and spontaneous motor behavior tests. PL significantly exhibited anti-depression like activity and cognitive enhancing effects, in comparison to the control groups after the first dose $(p<0.01)$ and the effects could be maintained throughout the period of study. Quantitative analysis of the brain homogenates by HPLC indicated that piperine, delivered either orally or nasally, distributed to the hippocampus at a higher extent than the cortex and that the time to peak concentration of nasal PL was shorter than for the oral piperine. Intranasal PL was, thus, potential in delivery of piperine, at a low dose, to exert its antidepressant and cognitive enhancing activities.
\end{abstract}

Keywords: Piperine Liposome; Anti-Depression Activity; Cognitive Enhancement Effect; Intranasal Route

\section{INTRODUCTION}

Piperine, a major alkaloid of black pepper (Piper nigrum Linn.) and long pepper (Piper longum Linn.), has been used in folk medicine for the treatment of various diseases, including seizure disorders [1-3]. Pharmacological studies have shown that piperine possesses various activities including anti-inflammatory and analgesic [4], anticonvulsant [5], anti-ulcer [6], anti-depressant [1], cytoprotection, antioxidant [7] and cognitive enhancing effect [8]. It inhibits monoamine oxi- dase (MAO) activity $[1,3,9]$ and increases the level of noradrenaline and serotonin in mouse brain [3], indicating its potential neurological benefits. Piperine could affect hippocampal neurogenesis in chronic mild stressed mice in which the level of brain-derived neurotrophic factor decreased. At a dose between 5 - 20 $\mathrm{mg} / \mathrm{kg}$ body weight given to cholinergic-deficient rats, induced by AF64A, piperine could attenuate the increase in lipid peroxidation and acetylcholinesterase activity [10]. Results from cell survival and the level of brain-derived neurotrophic factor in corticosterone-treated cultured hippocampal neurons confirm similar findings [11], yet the mechanism of actions of piperine on neuronal damage is still unknown.

The toxicity of piperine has been reported at an oral dose of 10 and $20 \mathrm{mg} / \mathrm{kg}$ body weight for 30 days $[12,13]$, particularly effects on male reproductive system and irritation to the skin and eye. $47 \%-64 \%$ of an oral dose of piperine would be subjected to the first-pass effect [14] with some distribution to other organs. To exert its neuropharmacological actions, high doses of piperine are thus required, yet, safety is also a major concern particularly in long-term use. Nanoparticles decrease toxicity of therapeutic drugs by overcoming the blood brain barrier (BBB) which limits brain delivery from the central compartment [15]. Lipid nanospheres which encapsulated piperine lowered its clearance rate and reduced its volume of distribution [16]. Thus, to deliver potentially toxic substances using nanosize particles might lower the doses as well as toxicity when compared to conventional dosage forms.

Liposomes could be prepared into nanosize vesicles $(20-100 \mathrm{~nm})$ formed by phospholipids and similar amphipathic lipids. Lipid bilayers of liposomes are similar in structure to those found in living cell membranes and can carry lipophilic substances such as 
drugs within these layers in the same way as cell membranes. This would facilitate drug transport and release across the BBB. A nerve growth factor, encapsulated in 100-nm liposomes, was protected from enzyme degradation in vivo and enhanced permeability across the BBB [17]. Proteins or peptide drugs could be delivered to the endothelial cells of brain capillary with a specific target to the lysosomes by encapsulation in liposomes [18].

Nasal administration, bypassing the BBB [19], offers a potential direct transfer of substances from the olfactory mucosa along the olfactory pathway to the central nervous system [20,21]. Drug absorption along the olfactory membrane, dependent on the characteristics of the compounds, is possible for systemic effect due to high degree of vascularization of the nasal mucosa and neuronal cells exposure which may facilitate transportation to the cerebrospinal fluid [22]. Hydrophobic compounds might diffuse or retrograde transport along the axon to the olfactory bulbs and diffuse to the rest of the brain. This could be relatively slow and ineffective in terms of drug usage. Hydrophilic compounds might transport into the cerebrospinal fluid via the subarachnoid space which extends along the olfactory nerves to the basolateral side of the olfactory epithelium. The intranasal route of several drugs was comparable to parenteral administration [23-25] with rapid brain delivery and first-pass avoidance [26]. Limitations include hydrophilic, labile and irritating compounds. Due to physiological limitation, the volume to be instilled into the nasal cavity for optimal effect is suggested to be less than $0.25 \mathrm{ml}$. Also, bioadhesive materials are suggested to overcome rapid mucociliary clearance which could decrease absorption [24,27-28]. Thus, this manuscript investigates piperine-encapsulated liposomes for brain delivery with polymers used to retain the liposomal dispersion inside the nasal cavity. Dose reduction when a compound is given in liposomal forms is expected $[15,29]$, thus, for piperine, a lower dose was estimated and proposed for the first time in this study.

To study the effect of piperine delivery on the antidepressant and cognitive actions in animals, several recognized and well-established tests using animal models were used. The Morris water maze (MWM) test is an effective model for investigation of cognitive effects because the deficits in (hidden-plat-form) MWM performance could relate to the intellectual decline in human Alzheimer patients [30]. The forced swimming test is used in screening of anti-depressant activity [31]. Stereotyped behaviors, a group of repetitive and invariant behaviors are used to indicate psychological well-being related to the living environment, as follows: grooming indicating a hygienic activity of predictable sequence of activities directed to the animal body surface [32] which involves the function of dopamine receptors, especially D1 and D2 [33,34]; rearing behavior, expressed by rising hind legs without front paws touching any surface, associated with the selective elevation of striatal glutamate (Glu) and aspartate (Asp) concentrations [35] and dopamine [36]; licking behavior, involved the alteration of the transmitter function such as dopamine [37] and serotonin [38].

From our previous study [8], oral piperine has shown to be effective as a cognitive enhancing compound. Oral administration of piperine involves first-pass metabolism and relatively high doses which could lead to adverse side effects or other undesired consequences. This study intended to develop an intranasal delivery of piperine using a form of encapsulation in liposomes and study depression and cognition effects in animal models by comparison to the conventional oral piperine. The intranasal dose of piperine was proposed. It was hypothesized that both treatments would deliver piperine to exert the desire activities in the brain.

\section{MATERIALS AND METHODS}

\subsection{Materials}

High-purity egg L- $\alpha$-phosphatidylcholine (EPC), piperine and potassium chloride (Sigma-Aldrich, Germany), cholesterol (chol, Sigma-Aldrich, Japan), potassium phosphate monobasic, $\left(\mathrm{KH}_{2} \mathrm{PO}_{4}\right.$, Sigma-Aldrich, U.S.A.) and polyethylene glycol 1000 (PEG, Sigma, Spain) were used. Analytical or HPLC grade solvents, such as chloroform, absolute ethanol, acetonitrile and orthophosphoric acid, were from BDH Laboratory Supplies (Poole, England). Heparin sodium was obtained from LEO Pharmaceutical Products (Denmark). Deionized water was used throughout this study. All chemicals were used as received.

\subsection{Piperine-Encapsulated Liposomes}

Thin film hydration with extrusion [39-41] was the method of choice in the preparation of piperine-encapsulated liposomes (PL) used in this study. In brief, a lipid film was formed during degassing and moderate rotation at $60^{\circ} \mathrm{C}$ using a 1:2 molar ratio of EPC and chol in the presence of piperine and chloroform. Subsequently, it was hydrated with water (about $60^{\circ} \mathrm{C}$ ), sonicated, extruded ( $\mathrm{Li}-$ posoFast, Avestin, Canada) twice through 0.2- $\mu \mathrm{m}$ and then $0.1 \mu \mathrm{m}$ polycarbonate membranes (Avestin, Canada) and mixed with propylene glycol and PEG. The PL, contained $0.6 \mathrm{mg} / \mathrm{ml}$ of piperine, were freshly-prepared using aseptic technique throughout the study. Transmission electron microscope (TEM, Joel JEM 2010, JEOL Co. Ltd., Tokyo, Japan) was used to view the PL. Percentage encapsulation efficiency $(\% \mathrm{EE})$ of piperine in the 
liposomes was estimated by the following Eq.1:

$$
\% E E=\left(\frac{C_{\text {tol }}-C_{\text {free }}}{C_{\text {tol }}}\right) \times 100
$$

where $E E$ is the entrapment efficiency, $C_{\text {tol }}$ is total quantity of added piperine and $C_{\text {free }}$ is quantity of free piperine detected from supernatant.

\subsection{Animal}

Adult male Wistar rats $(\mathrm{n}=8,180-220 \mathrm{~g}, 8$ weeks old $)$ were obtained from the National Animal Center, Nakorn Pathom, Thailand. Male rats were recommended because of the advantage in spatial learning [30]. Upon arrival, they were housed in standard metal cages (5 each) at $21^{\circ} \mathrm{C} \pm 1{ }^{\circ} \mathrm{C}$ and $65 \%$ relative humidity with a $10 \mathrm{~h}$ light $/ 14 \mathrm{~h}$ dark cycle and given standard chow and water ad libitum for the duration of the study. They were allowed 2 weeks to adapt to their environment before use in this experiment.

The rats were randomly assigned into four groups, i.e. oral piperine, intranasal PL, vehicle, and blank liposomes, containing eight animals per group in each experiment. Each animal was subjected to the behavioral assessment at a predetermined period. The experiments were conducted following the approved protocol by the Ethics Committee of the institute which minimized the number of animals used in the study, also attempts were made to minimize animal suffering in accordance with the internationally accepted principles for laboratory use and care of the European Community (EEC Directive of 1986; 86/609/EEC).

Prior to determine the dose of PL, estimation from the pharmacokinetic profile of piperine and preliminary study was thoroughly investigated. PL with a concentration of piperine of $0.6 \mathrm{mg} / \mathrm{ml}$ was administered to each rat once daily at dose of $48 \mathrm{ng} / \mathrm{kg}$ body weight by dropping into the right nasal cavity. During and for $20 \mathrm{sec}$ after the nasal drop, the rat was gently hold upright to ensure that the PL contacted the nasal mucosa and moved into the deeper part of the nasal cavity. Animals in conventional piperine group were subjected to intragastric administration of freshly-prepared piperine solution in propylene glycol once daily at a dose of $5 \mathrm{mg} / \mathrm{kg}$ body weight. Care was taken to conduct each experiment at the same period of the day to avoid deviations in animal behavior.

\subsection{Behavioral Assessments}

Each rat was assessed by Morris water maze (MWM) and forced swimming tests as well as daily spontaneous motor behavior assessment, as per the following details:

Morris water maze test: The Morris water maze test was used as a tool to determine cognitive function of the rats that were trained to memorize the location of the hidden platform in relation to its location. Animals with enhanced cognition would be demonstrated by a decrease in escape latency and an increase in retention time. The water maze consisted of a metal pool $(170 \mathrm{~cm}$ in diameter $\times 58 \mathrm{~cm}$ tall) filled with tap water $\left(25^{\circ} \mathrm{C}, 40 \mathrm{~cm}\right.$ deep) divided into four quadrants. In the center of one quadrant was a removable escape platform which was placed below the water level. The top of the water was covered with nontoxic milk powder. The pool was divided into four quadrants (NE, NW, SE, and SW) by two imaginary lines crossing the center of the pool. For each animal, the location of invisible platform was placed at the center of one quadrant and remained there throughout training. The rats must memorize the platform location in relation to various environmental cues because there was nothing directly showing the location of the escape platform in or outside the pool. Therefore, the placement of the water tank and platform were the same in all acquisition trials. Each rat was gently placed in the water facing the wall of the pool from one of the four starting points (N, E, S, or W) along the perimeter of the pool, and the animal was allowed to swim until it found and climbed onto the platform. During the training session, the rat was gently placed on the platform by experimenter if it could not reach the platform within 60 sec. In either case, the subject was left on the platform for $15 \mathrm{sec}$ and then removed from the pool. The time for animals to climb onto the hidden platform was recorded as escape latency or acquisition time. In order to determine the capability of the animals to retrieve and retain information, the platform was removed $24 \mathrm{~h}$ later and the rat was released into the quadrant diagonally opposite to that which contained the platform. Time spent in the region that previously contained the platform was recorded as retention time. In each trial, the animal was quickly dried with a towel before being returned to the cage.

Preliminary studies were conducted to organize the series of each test in the same pattern and timing. Right after the dosage administration, each set was subjected to the test within $30 \mathrm{~min}$. This was controlled throughout the study.

Forced swimming test: In order to assess the anti-depressant activity of the substance, the modified Porsolt test [42], a valid animal model for testing depression $[31,43]$, was conducted. Animals with depression would demonstrate an increase in immobility and also a decrease in active behavior (swimming, moving or climbing). Each rat was subjected to the behavior test, before and at predetermined period after the treatment, during a $5 \mathrm{~min}$ session in water, contained in a cylindrical glass aquarium ( $22 \mathrm{~cm}$ in diameter, $40 \mathrm{~cm}$ in height), at a depth of 
$20 \mathrm{~cm}$ and $25^{\circ} \mathrm{C} \pm 1^{\circ} \mathrm{C}$. The same trained observer, blinded from the intervention given to each rat, timed and recorded the durations that each rat spent on immobility, swimming and climbing throughout the 5 min session. The rats were considered immobile when neither of the hind leg was moving; the rats were slightly hunched forward. The total duration of immobility was measured during the 5-minutes test. Upon removal from the water, rats were towel-dried and finally returned to their home cage.

Spontaneous motor behavior assessment: In order to assure that anti-depression like activity and cognitive enhancing effect which were determined by various tests mentioned earlier were not false positive due to the effect of piperine on motor behavior, we also determined the effect of piperine on the spontaneous locomotor activity. All animals were assessed for spontaneous motor behavior including grooming and rearing licking behavior [44] for $5 \mathrm{~min}$. The performance was determined $60 \mathrm{~min}$ after substance administration.

\subsection{Quantitative Analysis of Piperine in the Brain Homogenate by HPLC}

The HPLC system: An HPLC with a UV/VIS detector (Perkin Elmer 200 IC, U.S.A.), a C18 column (4.6 mm $\times$ $250 \mathrm{~mm}, 5 \mu \mathrm{m}$, Theale Reading, Berks, U.K.) and Data Apex Charity ${ }^{\mathrm{TM}}$ Lite software was used. A mobile phase consisting of 1:1 of acetonitrile in $0.01 \mathrm{M} \mathrm{KH}_{2} \mathrm{PO}_{4}$ and adjusted to $\mathrm{pH} 4.5$ using orthophosphoric acid was used at a flow rate of $1.0 \mathrm{ml} / \mathrm{min}$, and detection was at $340 \mathrm{~nm}$. Piperine standards were freshly-prepared by diluting from a stock solution in absolute ethanol to a concentration range of $2-100 \mathrm{ng} / \mathrm{ml}$ with mobile phase. The standards showed good linear correlation and accuracy with an average $\% \mathrm{CV}$ of less than $15 \%$.

Brain homogenate: The cortex and hippocampus parts of the brain of each rat were isolated after it was anaesthetized and sacrificed. Each brain tissue was homogenized with $0.2 \mathrm{ml}$ of $1.15 \% \mathrm{KCl}$ [45]. The homogenate was then thoroughly mixed with $0.3 \mathrm{ml}$ of the HPLC mobile phase and centrifuged at 10,000 rpm for 30 min (Spectrafuge, U.S.A.). The supernatant was separated for HPLC analysis. Each data was obtained from three individuals with triplicate analyses from each.

\subsection{Statistical Analysis}

Data are presented as means \pm S.E.M. Statistical analysis was performed by one-way analysis of variance. A probability value of less than 0.05 was considered significant.

\section{RESULTS AND DISCUSSION}

Figures 1, 2 and 3 demonstrate that the results obtained from the rats treated with the vehicle, composed of pro-

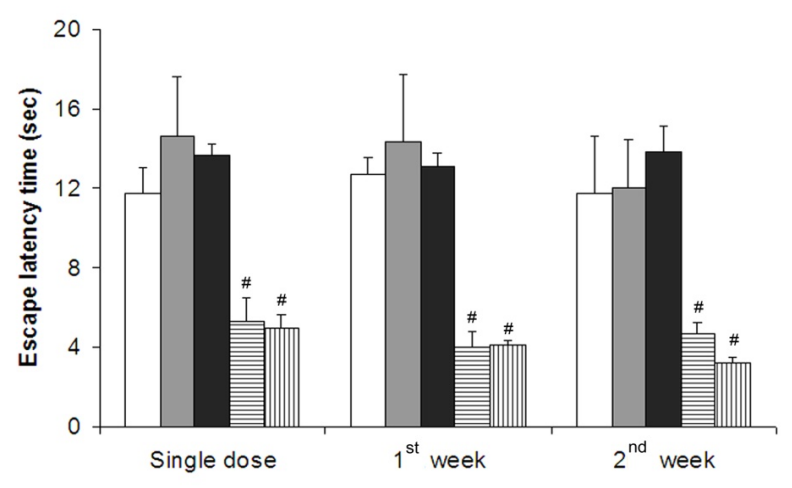

(a) Escape latency time

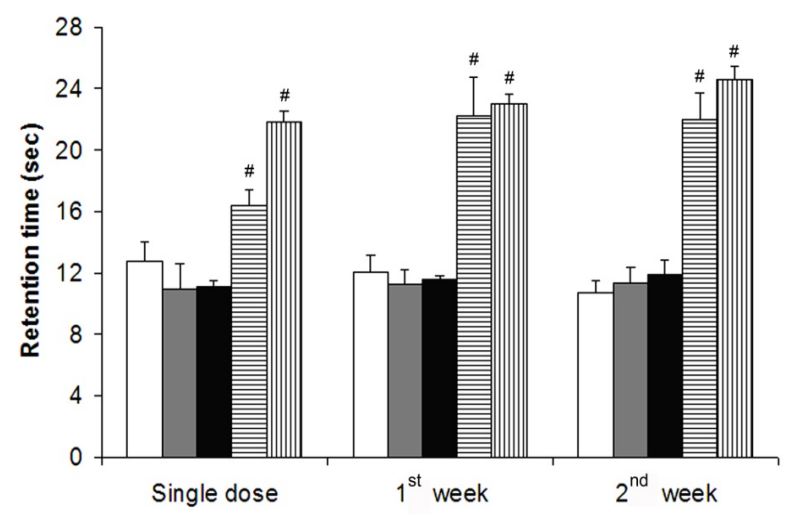

(b) Retention time

Figure 1. Morris water maze test of 5 groups of rats measured after a single dose or repeated once-daily doses at $1^{\text {st }}$ and $2^{\text {nd }}$ weeks; the control (clear column), treatment with vehicle (grey column), blank liposomes (black column), piperine $5 \mathrm{mg} / \mathrm{kg}$ body weight (horizontal stripes column) and piperine-encapsulated liposomes (vertical stripes column), each being a mean \pm S.E.M. $(n=8$ each $),{ }^{\#} p<0.001$ compared with vehicle or blank liposomes.

pylene glycol, PEG and water, or the blank liposomes, composed of EPC, chol, and the vehicle, used in this study were not significantly different from the results obtained from the relevant control groups ( $p>0.05$ all). The dose-dependent toxicity of piperine has been well reported, and thus, the oral dose of piperine was determined by following that reported by Wattanathorn et al. (2008) [8]. The intranasal dose was then proposed by estimation from its oral dose and pharmacokinetic profile reported earlier [8], based on minimal or none first-pass metabolism involvement on nasal absorption.

Spatial memory of the rats was assessed by MWM test and is shown in Figure 1(a) escape latency and 1(b) retention time. A 3-fold decrease in average escape latencies was observed, i.e. from $14.7 \pm 2.9$ to $5.4 \pm 1.2$ and $13.7 \pm 0.6$ to $4.9 \pm 0.7 \mathrm{sec}$ after single doses of oral 


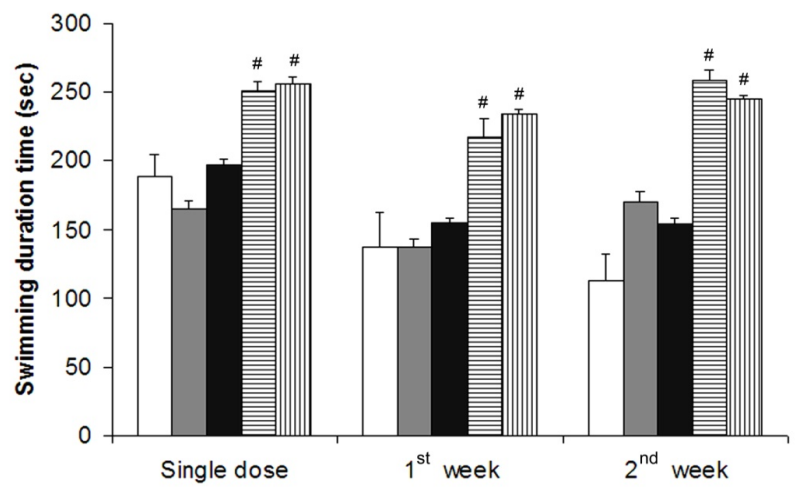

(a) Swimming duration

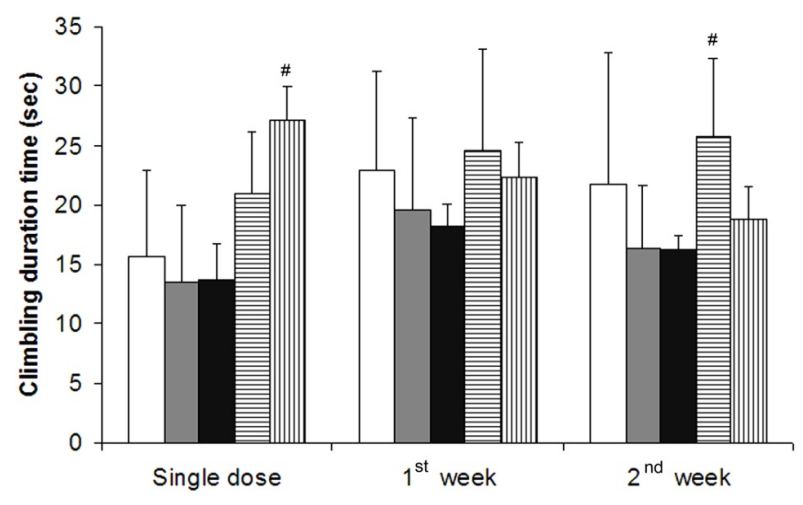

(b) Climbing duration

Figure 2. Forced swimming test of 5 groups of rats measured after a single dose or repeated once-daily doses at $1^{\text {st }}$ and $2^{\text {nd }}$ weeks; the control (clear), treatment with vehicle (grey), blank liposomes (dark), piperine $5 \mathrm{mg} / \mathrm{kg}$ body weight (horizontal stripes) and piperine-encapsulated liposomes (vertical stripes), each being a mean \pm S.E.M. $\left(\mathrm{n}=8\right.$ each), ${ }^{\#} p<0.001$ compared with vehicle or blank liposomes.

piperine and intranasal PL, respectively. This pattern of reduction was also observed after repeated daily doses for 1 and 2 weeks. Figure 1(b) showed an increase in the retention time, from $10.9 \pm 1.7$ to $16.4 \pm 1.1$ and 11.1 \pm 0.5 to $21.8 \pm 0.7 \mathrm{sec}$ after single doses of oral piperine and intranasal PL, respectively. Both results, escape latency reduction and retention time increase, were significantly different $(p<0.001)$ from the control, vehicle and blank liposome groups. The results of the oral piperine group and intranasal PL group, however, were not statistically different $(p>0.05)$. These indicate that oral piperine and intranasal PL enhanced cognitive function in the rats when compared to the non-treatment groups.

Forced swimming is a behavioral test used frequently to evaluate the potential efficacy of prospective antidepressant drugs in rats or mice [42]. Its principle lies on the fact that, while forced swimming, a test compound exerts its antidepressant by decreasing immobility time

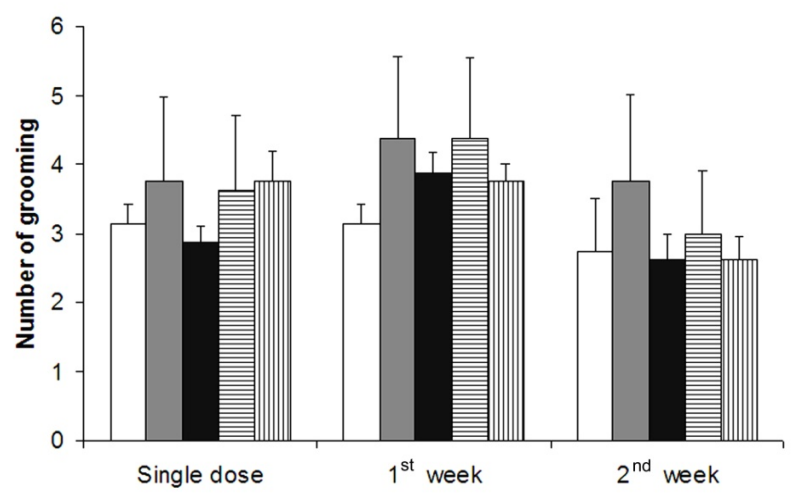

(a) Grooming behavior

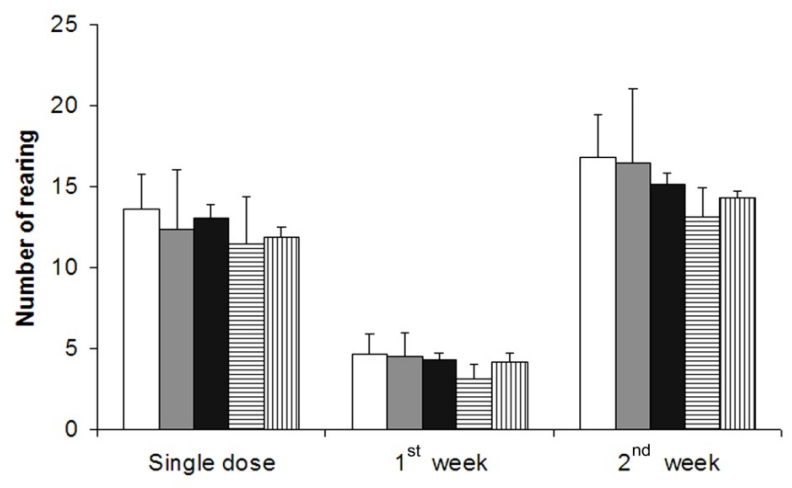

(b) Rearing behavior

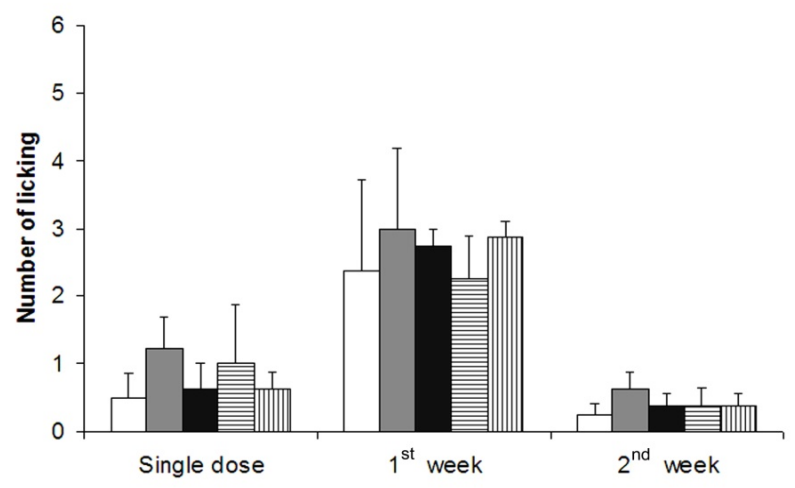

(c) Licking behavior

Figure 3. Spontaneous motor behavior test of 5 groups of rats measured after a single dose or repeated once-daily doses at $1^{\text {st }}$ and $2^{\text {nd }}$ weeks; the control (clear column), treatment with vehicle (grey column), blank liposomes (black column), oral piperine $5 \mathrm{mg} / \mathrm{kg}$ body weight (horizontal stripes column) and piperine-encapsulated liposomes (vertical stripes column), each being a mean \pm S.E.M. $(n=8$ each $)$.

and increasing active behaviors, which should be consistent with a therapeutic effect of increasing escape-directed activities [46]. Immersion of rodents in the water for an extended period of time produces a characteristic behavior called immobility, in which the rat makes only those movements necessary to keep its head above water. 
Figures 2(a) and 2(b) demonstrate that the PL treated rats significantly enhanced behavioral mobility, shown as the swimming times $(p<0.001)$, and climbing times $(p<0.01)$, after single dose of administration, 1 and 2 weeks of treatments. Oral piperine also gave similar results. Lee et al. [1] using tail suspension test in mice to investigate the effect of piperine on monoamine oxidase activity, reported that piperine decreased the immobility time in a dose-dependent manner at doses of 3 and $9 \mathrm{mg} / \mathrm{kg}$ body weight when given by intra-peritoneal injection. Because antidepressant activity of a compound usually enhances behavioral mobility [47], our results suggest that piperine, regardless of the delivery routes and dosage, inhibited depression-like behaviors in rats which were assessed by forced swimming test.

In order to prove that the reduction of immobility time in the force swimming test did not associate with a non-specific increase in locomotor activity or even decrease motor behavior in open field tests [31], we investigated the spontaneous motor activity induced by piperine in a separate experiment. The results were shown in Figure 3. In this study, both forms of piperine and the vehicles at doses which produced an antidepressant-like effect, did not significantly change spontaneous motor behavior including grooming, licking and rearing behavior ( $p>0.05$, all). This result demonstrated that the antidepressant-like effect of piperine in the MWM test was not based on any stimulation of locomotor activity.

Since the spontaneous motor behaviors of the test animals did not indicate that piperine affected any motor function of the brain, it affirmed that the anti-depression like activity and cognitive enhancing effects which were determined by force swimming and MWM tests were not affected in the presence of piperine at these doses. Piperine from the oral liquid and the intranasal liposomes, thus, has been shown to exert anti-depression and cognitive enhancing effects.

Figure 4 illustrates PLs which were small round particles surrounding with clusters of polymeric coating. By observation, the size of the liposomes with polymeric coating was about $100 \mathrm{~nm}$. The encapsulation efficiency of the liposomes, estimated by $\% \mathrm{EE}$ from 4 batches of preparations, was found to be $65 \pm 3.6 \%$. This indicates a moderate encapsulation which could be due to the hydrophobic characteristics of piperine. Coating with PEG was intended to enhance viscosity as well as assist the adherence of liposomal particles to the nasal mucosa. This might have facilitated the intranasal absorption of piperine into the brain and effectively showed activities at a daily dose of $48 \mathrm{ng} / \mathrm{kg}$ body weight.

Figure 5 compares the piperine content time course of two delivery systems in two portions of the brain issues, namely the hippocampus and the cortex. The content of

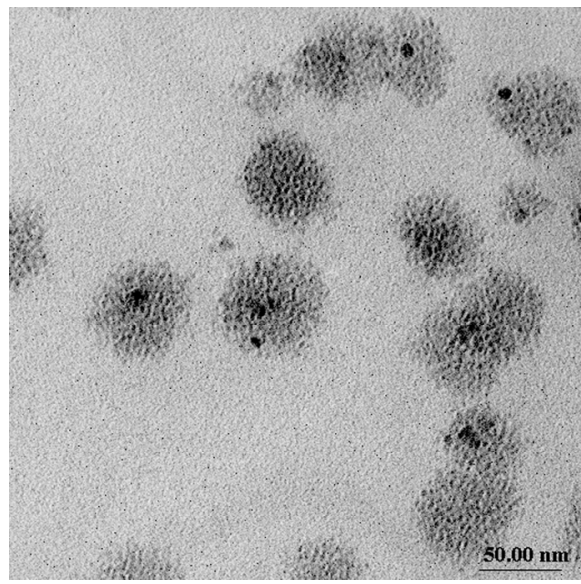

Figure 4. TEM photograph of piperine-encap sulated liposomes with PEG coating.

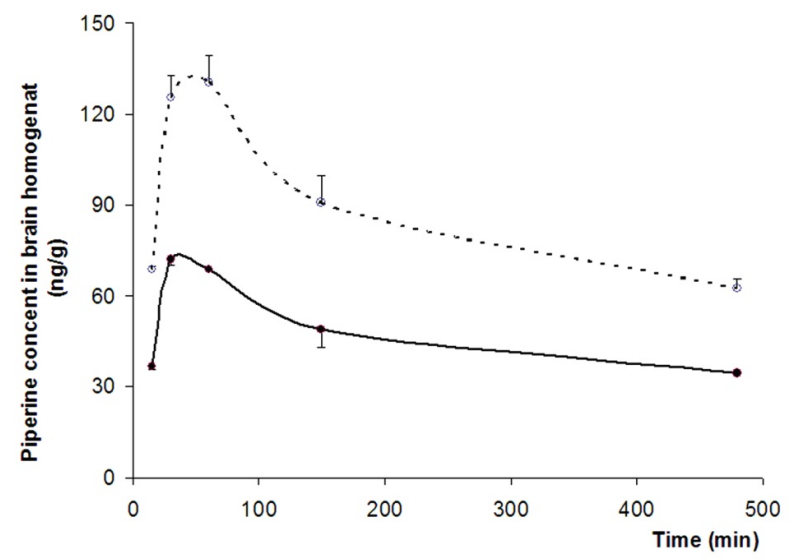

(a)

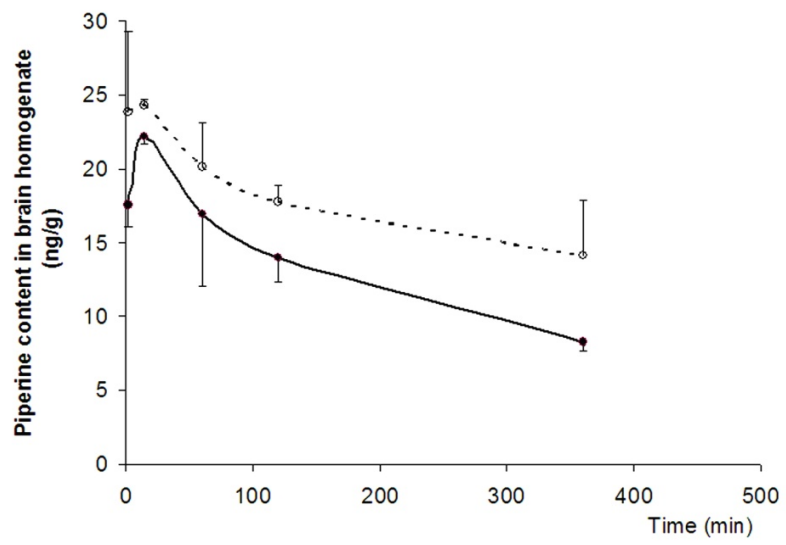

(b)

Figure 5. Piperine content extracted after administration of (a) oral piperine $(5 \mathrm{mg} / \mathrm{kg}$ body weight) and (b) nasal piperine liposomes $(0.048 \mathrm{mg} / \mathrm{kg}$ body weight $)$ at time interval from the brain tissues: hippocampus (--o--) and cortex (-๑-). The error bars represent S.E.M. $(\mathrm{n}=3$ each $)$.

piperine extracted after administration of oral piperine showed higher levels than nasal piperine liposomes. This 
was anticipated as the oral dose was about 100 times higher. The duration required to reach maximum brain levels after oral doses was about 40 - 50 min while that after nasal doses being $15 \mathrm{~min}$. Intranasal administration of piperine from this liposome formulation could rapidly deliver piperine into the brain although at lower levels than the oral solution. Both delivery systems suggested uneven distribution of piperine in different brain tissues, particularly the hippocampus which was the preference for piperine intake. The results support the behavioral assessment that piperine exerted its action within the brain and in the hippocampus, in particular. A dose-response study was not one of our objectives but our findings lead to imply that the dose needed for the brain activities of piperine might be less than that used in the oral doses. However, this needs further evidence.

In comparison to the previous study of Wattanathorn et al. [8] which employed an oral dose of $5 \mathrm{mg} / \mathrm{kg}$ body weight, this study showed a reduction of dose used to obtain similar outcome. Piperine, effective as an anti-depressant drug and cognitive enhancing drug, delivered intranasally in a liposomal encapsulation tends to be lower dose than from an oral suspension by about 200 times. Also, both anti-depression like and cognitiveenhancing effects were significantly improved after the first dose. This emphasizes an importance of the delivery form of piperine to exert its neurological actions in the brain.

Lee et al. [1] demonstrated that piperine could reversibly inhibited mouse brain MAO-A and MAO-B activities in a dose-dependent manner at i.p. doses of 3 and $9 \mathrm{mg} / \mathrm{kg}$ body weight. Its activity could be comparable to that of fluoxetine, a reference antidepressant agent, at $10 \mathrm{mg} / \mathrm{kg}$, i.p., and thus, conclude that the antidepressant-like effect of piperine may be mediated through the enhancement of serotonergic and norepineph-rinegic neurotransmission, as well as by dopaminergic neuro-transmission. However, the mechanism involved in the observed antidepressant activity is not elucidated. This study confirms its antidepressant activity at a lower dose when administered by intranasal PL. It is very interesting to that the anti-depression like activity and cognitive enhancing effect of piperine liposome are comparable to the piperine conventional form [8]. Piperine liposomes could be reproducibly prepared from $\mathrm{EPC} / \mathrm{chol}$ at a ratio of 2:1 and showed antidepressant and cognitive enhancing effect. The advantages of having a particle size in nanoscale, entrapment efficiency of about $60 \%$, are that the liposomes might permeate through the olfactory epithelium and release piperine to exert its actions.

The anti-depression and cognitive enhancing effects had previously shown to be associated with the alteration of various neurotransmitters including serotonin $[48,49]$ and acetylcholine [50] respectively. Intranasal administration, coupled with liposomal encapsulation facilitated the delivery of piperine via the olfactory pathway and might permeate and diffuse into the cerebrospinal fluid to exert its anti-depression and cognitive enhancing effects by alteration of acetylcholine and serotonin [2] respectively.

The results lead us to conclude that intranasal piperine liposomes could deliver piperine to the brain and potentially benefit for novel treatment of certain neuropharmacological disorders. Also, its unpleasant odor was successfully masked and its brain delivery particularly to the hippocampus was very rapid.

\section{CONCLUSIONS}

Piperine liposomes, particle sizes of less than $100 \mathrm{~nm}$ with an entrapment efficiency of about $60 \%$, when administered intranasally, could deliver piperine to the brain. Its pungent unpleasant odor was masked with the liposomal encapsulation and the addition of polymers. Also, intranasal piperine liposomes were shown to deliver to the hippocampus at a faster rate and higher extent than the oral dose. Intranasal delivery of piperine from the liposomes could therefore reduce the dose of piperine intake while giving the similar cognitive and antidepressant effects as the oral dosage.

\section{ACKNOWLEDGEMENTS}

This study was partially supported by the National Research Council of Thailand and the Development of Nutraceuticals and Brain Plasticity Research Group Khon Kaen University, Khon Kaen 40002.

\section{REFERENCES}

[1] Lee, S.A., Hong, S.S., Han, X.B., Hwang, J.S., Oh, G.J., Lee, K.S., Lee , M.K., Hwang, B.Y. and Ro, J.S. (2005) Piperine from the fruits of Piper longum with inhibitory effect on monoamine oxidase and antidepressant-like activity. Chemical \& Pharmaceutical Bulletin (Tokyo), 53, 832-835. doi:10.1248/cpb.53.832

[2] Li, S., Wang, C., Wang, M., Li, W., Matsumoto, K. and Tang, Y. (2007) Antidepressant like effects of piperine in chronic mild stress treated mice and its possible mechanisms. Life Sciences, 80, 1373-1381.

doi:10.1016/j.lfs.2006.12.027

[3] Li, S., Wang, C., Li, W., Koike, K., Nikaido, T. and Wang, M.W. (2007) Antidepressant-like effects of piperine and its derivative, antiepilepsirine. Journal of Asian Natural Products Research, 9, 421-430. doi:10.1080/10286020500384302

[4] Gupta, S.K., Bansal, P., Bhardwaj, R.K. and Velpandian, T. (2000) Comparative antinociceptive, anti-inflammatory and toxicity profile of nimesulide vs nimesulide and piperine combination. Pharmacological Research, 41, 657-662. doi:10.1006/phrs.1999.0640

[5] D’Hooge, R., Pei, Y.Q., Raes, A., Lebrun, P., Van Bogaert, 
P.P. and De Deyn, P.P. (1996) Anticonvulsant activity of piperine on seizures induced by excitatory amino acid receptor agonists. Arzneimittelforschung, 46, 557-560.

[6] Bai, Y.F. and Xu, H. (2000) Protective action of piperine against experimental gastric ulcer. Acta Pharmacologica Sinica, 21, 357-359.

[7] Selvendiran, K., Singh, J.P., Krishnan, K.B. and Sakthisekaran, D. (2003) Cytoprotective effect of piperine against benzo[a]pyrene induced lung cancer with reference to lipid peroxidation and antioxidant system in Swiss albino mice. Fitoterapia, 74, 109-115. doi:10.1016/S0367-326X(02)00304-0

[8] Wattanathorn, J., Chonpathompikunlert, P., Muchimapura, S., Priprem, A. and Tankamnerdthai, O. (2008) Piperine, the potential functional food for mood and cognitive disorders. Food and Chemical Toxicology, 46, 3106-3110. doi:10.1016/j.fct.2008.06.014

[9] Kong, L.D., Cheng, C.H. and Tan, R.X. (2004) Inhibition of MAO A and B by some plant-derived alkaloids, phenols and anthraquinones. Journal of Ethnopharmacology, 91, 351-355. doi:10.1016/j.jep.2004.01.013

[10] Chonpathompikunlert, P., Wattanathorn, J. and Muchimapura, S. (2010) Piperine, the main alkaloid of Thai black pepper, protects against neurodegeneration and cognitive impairment in animal model of cognitive deficit like condition of Alzheimer's disease. Food and Chemical Toxicology, 48, 798-802. doi:10.1016/j.fct.2009.12.009

[11] Duman, R.S., Malberg, J., Nakagawa, S. and D'Sa, C. (2000) Neuronal plasticity and survival in mood disorders. Biological Psychiatry, 48, 732-739. doi:10.1016/S0006-3223(00)00935-5

[12] Daware, M.B., Mujumdar, A.M. and Ghaskadbi, S. (2000) Reproductive toxicity of piperine in Swiss albino mice. Planta Medica, 66, 231-236. doi:10.1055/s-2000-8560

[13] Malini, T., Manimaran, R.R., Arunakaran, J., Aruldhas, M.M. and Govindarajulu, P. (1999) Effects of piperine on testis of albino rats. Journal of Ethnopharmacology, 64, 219-225. doi:10.1016/S0378-8741(98)00128-7

[14] Bhat, B.G. and Chandrasekhara, N. (1986) Studies on the metabolism of piperine: Absorption, tissue distribution and excretion of urinary conjugates in rats. Toxicology, 40, 83-92. doi:10.1016/0300-483X(86)90048-X

[15] Jain, K.K. (2007) Nanobiotechnology-Based Drug Delivery to the Central Nervous System. Neurodegenerative Diseases, 4, 287-291. doi:10.1159/000101884

[16] Veerareddy, P.R. and Vobalaboina, V. (2008) Pharmacokinetics and tissue distribution of piperine lipid nanospheres. Pharmazie, 63, 352-355.

[17] Xie, Y., Ye, L., Zhang, X., Cui, W., Lou, J., Naqai, T. and Hou, X. (2005) Transport of nerve growth factor encapsulated into liposomes across the blood-brain barrier: in vitro and in vivo studies. Journal of Controlled Release, 105, 106-119. doi:10.1016/j.jconrel.2005.03.005

[18] Visser, C.C., Stevanovic, S., Voorwinden, L.H., van Bloois, L., Gaillard, P.J., Danhof, M., Crommelin, D.J. and de Boer, A.G. (2005) Targeting liposomes with protein drugs to the blood-brain barrier in vitro. European Journal of Pharmaceutical Sciences, 25, 299-305.

[19] Romeo, V.D., de Meireles, J., Sileno, A.P., Pimplaskar, H.K. and Behl, C.R. (1998) Effects of physicochemical properties and other factors on systemic nasal drug de- livery. Advance Drug Delivery Reviews, 29, 89-116. doi:10.1016/S0169-409X(97)00063-X

[20] Illum, L. (2000) Transport of drugs from the nasal cavity to the central nervous system. European Journal of Pharmaceutical Sciences, 11, 1-18. doi:10.1016/S0928-0987(00)00087-7

[21] Mathison, S., Nagilla, R. and Kompella, U.B. (1998) Nasal route for direct delivery of solutes to the central nervous system: Fact or fiction? Journal of Drug Targeting, 5, 415-441. doi:10.3109/10611869808997870

[22] Frey, W.H., Liu, J., Chen, X., Thorne, R.G., Fawcett, J.R., Ala, T.A. and Rahman, Y.E. (1997) Delivery of 125I-NGF to the brain via the olfactory route. Drug Delivery, 4, 87-92. doi:10.3109/10717549709051878

[23] Dyer, A.M., Hinchcliffe, M., Watts, P., Castile, J., Jabbal-Gill, I., Nankervis, R., Smith, A. and Illum, L. (2002) Nasal delivery of insulin using novel chitosan based formulations: A comparative study in two animal models between simple chitosan formulations and chitosan nanoparticle. Pharmaceutical Research, 19, 998-1008. doi:10.1023/A:1016418523014

[24] Illum, L., Furraj, N., Critcheley, H. and Davis, S.S. (1988) Nasal administration of gentamicin using a novel microsphere delivery system. International Journal of Pharmaceutics, 46, 261-265. doi:10.1016/0378-5173(88)90087-7

[25] Vyas, S.P., Goswami, S.K. and Ranjit, S. (1995) Liposomes based nasal delivery system of nifedipine: Development and characterization. International Journal of Pharmaceutics, 118, 23-30. doi:10.1016/0378-5173(94)00296-H

[26] Hussain, A.A. (1998) Intranasal drug delivery. Advance Drug Delivery Reviews, 29, 39-49. doi:10.1016/0378-5173(94)00296-H

[27] Illum, L., Jørgensen, H., Bisgaard, H., Krogsgaard, O. and Rossing, N. (1987) Bioadhesive microsphere as a potential nasal drug delivery system. International Journal of Pharmaceutics, 39, 189-199. doi:10.1016/0378-5173(87)90216-X

[28] Vyas, S.P., Bhatnagar, S., Gogol, P.J. and Jain, N.K. (1991) Preparation and characterization of HSA propranolol microspheres for nasal administration. International Journal of Pharmaceutics, 69, 5-12. doi:10.1016/0378-5173(91)90081-X

[29] Kreuter, J. (2001) Nanoparticulate systems for brain delivery of drugs. Advance Drug Delivery Reviews, 47, 65-81. doi:10.1016/S0169-409X(00)00122-8

[30] D'Hooge, R. and de Deyn, P.P. (2001) Application of the Morris water maze in the study of learning and memory. Brain Research Reviews, 36, 60-90. doi:10.1016/S0165-0173(01)00067-4

[31] Detke, M.J., Rickels, M. and Lucki, I. (1995) Active behaviors in the rat forced swimming test differentially activated by serotonergic and noradrenergic antidepressants. Psychopharmacology (Berl), 121, 66-72. doi:10.1007/BF02245592

[32] Adan, R.A., Szklarczyk, A.W., Oosterom, J., Brakkee, J.H., Nijenhuis, W.A., Schaaper, W.M., Meloen, R.H. and Gispen, W.H. (1999) Characterization of melanocortin receptor ligands on cloned brain melanocortin receptors and on grooming behavior in the rat. European Journal of Pharmacology, 378, 249-258. 
doi:10.1016/S0014-2999(99)00465-3

[33] Murray, A.M. and Waddington, J.L. (1989) Behavioural indices of the interaction of clozapine with D1 and D2 dopamine receptors. British Journal of Pharmacology, 98, 814.

[34] Sobrian, S.K., Jones, B.L., Varghese, S. and Holson, R.R. (2003) Behavioral response profiles following drug challenge with dopamine receptor subtype agonists and antagonists in developing rat. Neurotoxicology and Teratology, 25, 311-328. doi:10.1016/S0892-0362(03)00009-6

[35] Presti, M.F., Watson, C.J., Kennedy, R.T., Yang, M. and Lewis, M.H. (2004) Behavior-related alterations of striatal neurochemistry in a mouse model of stereotyped movement disorder. Pharmacology Biochemistry and Behavior, 77, 501-507. doi:10.1016/j.pbb.2003.12.004

[36] Lodge, D.J. and Lawrence, A.J. (2003) The effect of isolation rearing on volitional ethanol consumption and central CCK/dopamine systems in Fawn-Hooded rats. Behavioural Brain Research, 141, 113-122. doi:10.1016/S0166-4328(02)00328-5

[37] Higgs, S. and Cooper, S.J. (2000) The effect of the dopamine D2 receptor antagonist raclopride on the pattern of licking microstructure induced by midazolam in the rat. European Journal of Pharmacology, 409, 73-80. doi:10.1016/S0014-2999(00)00802-5

[38] Asin, K.E., Davis, J.D. and Bednarz, L. (1992) Differential effects of serotonergic and catecholaminergic drugs on ingestive behavior. Psychopharmacology (Berl), 109, 415-421. doi:10.1007/BF02247717

[39] Guo, J., Ping, Q., Jiang, G., Huang, L. and Tong, Y. (2003) Chitosan-coated liposomes: Characterization and interaction with leuprolide. International Journal of Pharmaceutics, 260, 167-173. doi:10.1016/S0378-5173(03)00254-0

[40] Liang, M.T., Davies, N.M. and Toth, I. (2005) Encapsulation of lipopeptides within liposomes: Effect of number of lipid chains, chain length and method of liposome preparation. International Journal of Pharmaceutics, 301, 247-254. doi:10.1016/j.ijpharm.2005.06.010

[41] Priprem, A., Rahman, Y.E., Juhn, S.K., Lakkaraju, A. and Pituksuteepong, T. (1999) A liposome-encapsulated am- picillin. Mahidol Journal, 6, 55-57.

[42] Porsolt, P.D., Anton, G., Blavet, N. and Jalfre, M. (1978) Behavioural despair in rats: A new model sensitive to antidepressant treatments. European Journal of Pharmacology, 47, 379-391. doi:10.1016/0014-2999(78)90118-8

[43] Lucki, I. (1997) The forced swimming test as a model for core and component behavioral effects of antidepressant drugs. Behavioural Pharmacology, 8, 523-532. doi:10.1097/00008877-199711000-00010

[44] Mason, G.J. (1991) Stereotypies: A critical review. Animal Behaviour, 41, 1015-1037. doi:10.1016/S0003-3472(05)80640-2

[45] Ohkawa, H., Ohishi, N. and Yagi, K. (1979) Assay for lipid peroxides in animal tissues by thiobarbituric acid reaction. Analytical Biochemistry, 95, 351-358. doi:10.1016/0003-2697(79)90738-3

[46] Page, M.E., Brown, K. and Lucki, I. (2003) Simultaneous analyses of the neurochemical and behavioral effects of the norepinephrine reuptake inhibitor reboxetine in a rat model of antidepressant action. Psychopharmacology (Berl), 165, 194-201.

[47] Petit-Demouliere, B., Chenu, F. and Bourin, M. (2004) Forced swimming test in mice: A review of antidepressant activity. Psychopharmacology (Berl), 177, 245-255. doi:10.1007/s00213-004-2048-7

[48] Lucassen, P.J., Fuchs, E. and Czeh, B. (2004) Antidepressant treatment with tianeptine reduces apoptosis in the hippocampal dentate gyrus and temporal cortex. Biological Psychiatry, 55, 789-796. doi:10.1016/j.biopsych.2003.12.014

[49] Schloss, P. and Henn, F.A. (2004) New insights into the mechanisms of antidepressant therapy. Pharmacology \& Therapeutics, 102, 47-60. doi:10.1016/j.pharmthera.2004.02.001

[50] Ingkaninan, K., Temkitthawon, P., Chuenchom, K., Yuyaem, T. and Thongnoi, W. (2003) Screening for acetylcholinesterase inhibitory activity in plants used Thai traditional rejuvenation and neurotonic remedies. Journal of Ethonopharmacology, 89, 261-264. doi:10.1016/j.jep.2003.08.008 